\title{
Experimental Validations of Bandwidth Compressed Multicarrier Signals
}

\author{
(Invited Paper) \\ Tongyang $\mathrm{Xu}$ and Izzat Darwazeh

\begin{abstract}
Department of Electronic and Electrical Engineering, University College London, London, UK
Email: t.xu@ee.ucl.ac.uk, i.darwazeh@ucl.ac.uk
\end{abstract}

\begin{abstract}
We comprehensively summarize experimental validations ${ }^{1}$ of bandwidth compressed multicarrier waveforms for future $5^{\text {th }}$ generation (5G) applications. The proposed waveforms are derived from an existing non-orthogonal multicarrier concept termed spectrally efficient frequency division multiplexing (SEFDM) where sub-carriers are non-orthogonally packed at frequencies below the symbol rate. This improves the spectral efficiency at the cost of self-created inter carrier interference (ICI).

In this work, experiments are reported and testing is carried out in three scenarios including long term evolution (LTE)like wireless link; millimeter wave radio-over-fiber (RoF) link and optical fiber link. In the first scenario, for a given 25 MHz bandwidth, the SEFDM testbed can provide $70 \mathrm{Mbit} / \mathrm{s}$ gross data rate while only $50 \mathrm{Mbit} / \mathrm{s}$ can be achieved for an OFDM system occupying the same bandwidth. For the millimeter wave experiment, occupying a $1.125 \mathrm{GHz}$ bandwidth, the gross bit rate for OFDM is $2.25 \mathrm{Gbit} / \mathrm{s}$ and with $40 \%$ bandwidth compression, $3.75 \mathrm{Gbit} / \mathrm{s}$ can be achieved for SEFDM. Two experimental optical fiber links are described in this work; a $10 \mathrm{Gbit} / \mathrm{s}$ direct detection optical SEFDM system and a 24 Gbit/s coherent detection SEFDM system. The LTE-like signals and millimeter wave technologies are well suited to provide last mile communications to end users as both can support mobility in wireless environments. The lightwave signals delivered by optical fibers would offer higher data rates and support longhaul communications. The reported techniques, used individually or combined, would be of interest to future wireless system designers, where bandwidth saving is of importance, such as in 5G networks, aiming to provide high capacity and high mobility, simultaneously while saving spectrum.
\end{abstract}

Index Terms-Multicarrier, 5G, LTE, spectral efficiency, OFDM, SEFDM, non-orthogonal, waveform, carrier aggregation, millimeter wave, radio over fiber, fiber wireless, optical fiber.

\section{INTRODUCTION}

The exponentially increased demand for higher data rates in future $5 \mathrm{G}[6]$ networks is challenged by the limitations of existing technologies. Today's wireless communication systems mostly employ multicarrier transmission techniques due to their efficient spectrum utilization and resistance to multipath fading. The most featured multicarrier technique is orthogonal frequency division multiplexing (OFDM), which is currently the system of choice for many wired and wireless transmission systems [7]. Signals are carried on parallel orthogonally

\footnotetext{
${ }^{1}$ The work in this paper gathers recent experimental work reported by the authors in $[1][2][3][4][5]$.
}

978-1-5090-2185-7/16/\$31.00 (c)2016 IEEE overlapped sub-carriers with frequency separation equal to the symbol rate. As a prominent physical layer technique, OFDM is currently used in the downlink transmission system for the $4^{\text {th }}$ generation (4G) cellular system LTE [8].

In order to maintain the orthogonality property, the spectral efficiency of OFDM can not be improved further without changing modulation formats. To overcome the challenge, some emerging techniques such as massive multiple input multiple output (MIMO) (space domain) [9], millimeter wave (frequency domain) [10] and beamforming (power domain) [11] are combined with OFDM to address this challenge. In addition, some novel waveforms such as filterbank based multicarrier (FBMC) [12] and generalized frequency division multiplexing (GFDM) [13] have been investigated as such techniques offer low out-of-band power leakage. Thus, they are notably used in cognitive radio scenarios.

A new direction for waveform design is to compress signal bandwidth whilst maintaining the same transmission rate per sub-carrier. Conceptually, this is a non-orthogonal waveform technique termed SEFDM, which provides improved spectral efficiency by packing sub-carriers at frequency spacing below the symbol rate. The principle of SEFDM was derived based on Fast OFDM [14] and was proposed in 2003 [15]. An alternative technique that results in a similar non-orthogonal multicarrier signal is termed multistream faster than Nyquist (FTN) and this has been studied extensively since 2006 [16]. Detailed studies of these techniques and a related technique termed time frequency packing (TFP) [17] have been done and summarized recently in an IEEE Proceedings paper [18]. Interestingly, these techniques found their fundamental origin in a 1975 paper by Mazo [19], where the work argued that higher spectral efficiencies may be achieved by sampling at rates below the Nyquist rate. The information theoretic capacity of the above techniques has been addressed by several research groups [20][21][22]. Specifically, for SEFDM, since 2003 a number of researchers have worked on different aspects of SEFDM signals ranging from signal generation and reception through their practical implementations using state-of-the-art hardware devices.

4G networks have been commercially set up and they pave the way to the deployment of future $5 \mathrm{G}$ networks. A standalone technique may not be able to support future $5 \mathrm{G}$ networks. It is expected that future $5 \mathrm{G}$ wireless networks 
will be a mixture of different backhaul and radio access technologies. In terms of future $5 \mathrm{G}$ systems, SEFDM would offer significant bandwidth saving advantage if used either as an air interface technique or for backhauling signals over wireless and/or wired and fibre communication channels.

In this paper we summarize experimental work that demonstrates the benefits of SEFDM in radio access domain and backhaul domain. Specially we describe:

- An enhanced LTE-like SEFDM signal is experimentally evaluated in a carrier aggregation (CA) scenario [1] in a real world wireless testbed together with a realistic LTE multipath fading channel.

- A bandwidth compressed $60 \mathrm{GHz}$ millimeter wave signal [2][3] is designed and tested in a point-to-point indoor fiber wireless RoF experiment.

- Optical fiber signal transmission is operated in both direct [4] and coherent [5] detection testbeds for the validations of future backhaul connections.

The rest of the paper is organized as follows. Section II gives a description of the fundamentals of SEFDM including signal model, signal generation, channel estimation and signal detection. Section III describes the applications for future $5 \mathrm{G}$ radio access technologies including the compressed $\mathrm{CA}$ experiment utilizing an LTE-like SEFDM signal and the 60 $\mathrm{GHz}$ millimeter wave SEFDM experiment in an integrated fiber and wireless link. Section IV shows the experimental setup and measured results of the direct and coherent optical testbeds. Finally, Section V concludes the paper.

\section{FUnDAMENTALS OF SEFDM}

The deliberate violation of the orthogonality principle leads to the new SEFDM waveform as shown in Fig. 1. Both OFDM and SEFDM sub-carriers packing schemes are demonstrated for the purpose of comparison. On one hand, through orthogonal multiplexing, OFDM waveform is obtained by aggregating several sub-carriers; for example in LTE downlink each subcarrier is effectively loaded with a $15 \mathrm{ksymbol} / \mathrm{s}$ data and sub-carriers are arranged to have $15 \mathrm{kHz}$ frequency spacing [23]. On the other hand, SEFDM waveform is generated after non-orthogonally multiplexing sub-carriers with compressed frequency spacing smaller than $15 \mathrm{kHz}$, yet maintaining the data rate and thereby allowing a higher number of equally loaded sub-carriers within the same overall signal band. It should be noted that in the time-domain, both OFDM and SEFDM symbols are transmitted satisfying the Nyquist ISIfree criterion. Therefore, it is inferred that transmitting the same amount of data, SEFDM occupies smaller bandwidth than OFDM.

However, the bandwidth compressed signal improvement of spectral efficiency comes at the cost of increased complexity [24] at transmitting and receiving ends. This section firstly describes the basic SEFDM signal model and then presents specially designed algorithms for signal generation, channel estimation and signal detection.

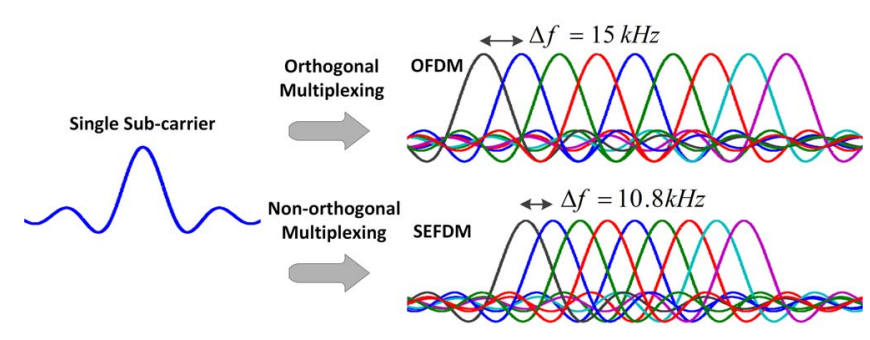

Figure 1. Fundamentals of SEFDM. The sub-carrier spacing is set to be $\Delta f=15 \mathrm{kHz}$, which is based on the LTE standard.

\section{A. Signal Model}

A discrete version of the SEFDM signal is mathematically expressed as

$$
X[k]=\frac{1}{\sqrt{Q}} \sum_{n=0}^{N-1} s_{n} \exp \left(\frac{j 2 \pi n k \alpha}{Q}\right)
$$

where $\alpha=\Delta f T$ is the bandwidth compression factor, $\Delta f$ is the sub-carrier spacing, $T$ is the period of one SEFDM symbol, $N$ is the number of sub-carriers, $Q=\rho N$ and $\rho \geq 1$ is the oversampling factor, $X[k]$ is the $k^{t h}$ time sample with $k=[0,1, \ldots, Q-1], \frac{1}{\sqrt{Q}}$ is a normalization scaling factor and $s_{n}$ is a QAM symbol modulated on the $n^{\text {th }}$ sub-carrier. Occupying the same number of sub-carriers, SEFDM can save $(1-\alpha) \times 100 \%$ of bandwidth compared to OFDM.

Due to the introduction of $\alpha$, adjacent sub-carriers are no longer orthogonal. Thus, SEFDM poses a great challenge in signal generation, channel estimation and signal detection since self-created ICI is introduced.

\section{B. Signal Generation}

According to (1), the SEFDM signal can not be directly generated using the typical IFFT algorithm, due to the nonorthogonal packed sub-carriers. Direct use of (1) results in high computational complexity. In order to use the highly efficient IFFT algorithm, some techniques have been studied and practically implemented in [25][26].

One approach is to pad zeros after each input vector (i.e. a vector of QAM symbols) to neutralize the effect of $\alpha$. The length of the new vector becomes $M=Q / \alpha$. Therefore, a single IFFT with extended length is feasible for SEFDM signal generation. However, one strict requirement is that $Q / \alpha$ has to be an integer and simultaneously a power of two, in other words $Q / \alpha \in 2^{\mathbb{N}^{+}}$, allowing for the IDFT to be implemented by means of the computationally efficient radix-2 IFFT.

Alternatively, a multiple IFFTs algorithm was designed that can effectively relax the requirement of $Q / \alpha$. The general idea is to interpolate zeros in each input vector instead of padding zeros. By setting $\alpha=b / c$ where $b, c \in 2^{\mathbb{N}^{+}}$, the length of the new input vector is extended to be $c Q$. The single long vector can be rearranged to $c$ parallel shorter vectors where an IFFT of length $Q$ is applied to each vector. It is inferred that an SEFDM signal can be generated using $c$ parallel shorter 
IFFTs. In other words, an SEFDM signal is composed of $c$ overlapped OFDM signals.

\section{Channel Estimation}

In a practical wireless system, due to the effect of multipath fading channel, imperfect timing synchronization and phase offset, channel estimation and compensation algorithms are required. For a typical OFDM system, a single-tap frequencydomain equalizer is applied. However, the compensation for SEFDM signals is challenged by the non-orthogonal overlapped sub-carriers. The loss of orthogonality in SEFDM greatly affects the accuracy of channel estimation. Thus the typical OFDM compensation approach is not directly applicable. Therefore, a time-domain compensation algorithm was designed to overcome the above challenge. The concept of SEFDM channel estimation and equalization was initially addressed in [27] and SEFDM time-domain channel compensation was comprehensively discussed in [28] where partial channel estimation (PCE) and full channel estimation (FCE) schemes are compared.

The basic concept of PCE is to modulate a subset of subcarriers, which are mutually orthogonal. Thus, the ICI effect can be removed leading to more accurate channel estimation. On the other hand, FCE is a general scheme in which pilot symbols are modulated on the entire sub-carriers.

\section{Signal Detection}

Signal detection is also challenged by the loss of orthogonality. The main purpose of the signal detection is to recover signals from the ICI. Several methods have been studied since the inception of SEFDM, utilizing optimum and sub-optimum techniques such as orthonormalization [29] and sphere decoding (SD) [30]. The SD was then used in a multiband system [31] to efficiently recover distorted signals in each band. Iterative detection was proposed in [32] and shown to enable the reduction of ICI using a combined feedforward and feedback structure. The feedforward loop aims to estimate symbols while the feedback loop is to remodel the ICI information on each symbol. After removing the interference, more reliable received symbols are fed to the loop until reaching a converged symbol estimation. It can be inferred that the remodelled inference information is crucial and it determines the accuracy of the interference cancellation and further affects the iteration performance.

The uncoded iterative detection scheme was studied in work [32] with limitations such as the bandwidth compression factor and the number of sub-carriers. An improved interference cancellation scheme termed Turbo equalization [33] can improve the reliability of interference modelling using error correction codes. In the Turbo-SEFDM system, soft information is exchanged between an inner and an outer decoder several times until a converged performance is obtained. By using this scheme, LTE-like signals with high bandwidth compression can be transmitted and recovered at the receiver side.

\section{ApPlichtions FOR Promising 5G}

At low frequencies, $\mathrm{CA}$ is a featured technique standardized in LTE-Advanced [34]. It offers a method to support a wider bandwidth by aggregating legacy fragmented frequency bands. Up to 5 component carriers (CCs) of $20 \mathrm{MHz}$ can be combined leading to a maximum $100 \mathrm{MHz}$ aggregated bandwidth. However, such a wide spectrum is not always available. Our proposal is to compress the bandwidth of each $\mathrm{CC}$ using SEFDM signals. This provides benefits such as bandwidth saving for a given number of CCs or more aggregated CCs in a given bandwidth. Spectral efficiency will be improved in both cases.

The congestion of existing spectrum makes it difficult to accommodate new wireless services requiring support for ultra wideband signals. An alternative way is to use higher frequencies such as $60 \mathrm{GHz}$ millimeter wave frequency since the available spectrum is substantially rich. The $7 \mathrm{GHz}$ [35] wide contiguous spectrum ranging from $57 \mathrm{GHz}$ to $64 \mathrm{GHz}$ can be utilized for applications requiring high transmission bandwidth. It should be mentioned that the $7 \mathrm{GHz}$ bandwidth limit can be advantageously utilized by using SEFDM signals. The millimeter wave experiment is based on fiber supported signal delivery to antenna units; i.e a RoF system. A low frequency RoF system (not discussed here) was reported by the authors and operated in the $2 \mathrm{GHz}$ band. Readers are referred to [36] for further details.

Henceforth, this section reports measurements of carrier aggregated SEFDM signal transmission in an existing LTE spectrum in the $2 \mathrm{GHz}$ band as well as measurements of single band SEFDM signal transmission in the $60 \mathrm{GHz}$ millimeter wave band for future spectrally efficient communications.

\section{A. Carrier Aggregation}

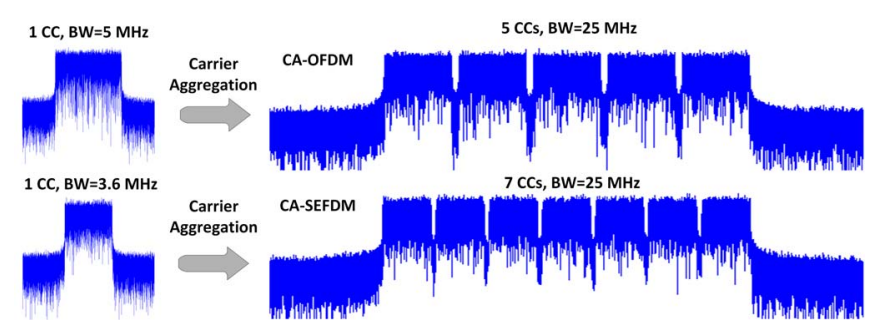

Figure 2. Carrier aggregation scenarios for both OFDM and SEFDM.

The basic principle of CA-SEFDM is shown in Fig. 2. An LTE standard signal is adopted for the OFDM scenario. At the first stage, the single band spectra for OFDM and SEFDM are illustrated. It is evident that the SEFDM signal bandwidth is compressed. This bandwidth saving benefit provides an approach to enhance the CA scenario and the way for the enhancement is shown at the second stage. It is apparent that the spectral efficiency of CA-SEFDM is increased by aggregating more $\mathrm{CCs}$ while maintaining the same data rate per sub-carrier. Therefore, for the same bandwidth occupation, CA-SEFDM offers a higher throughput than CA-OFDM. 


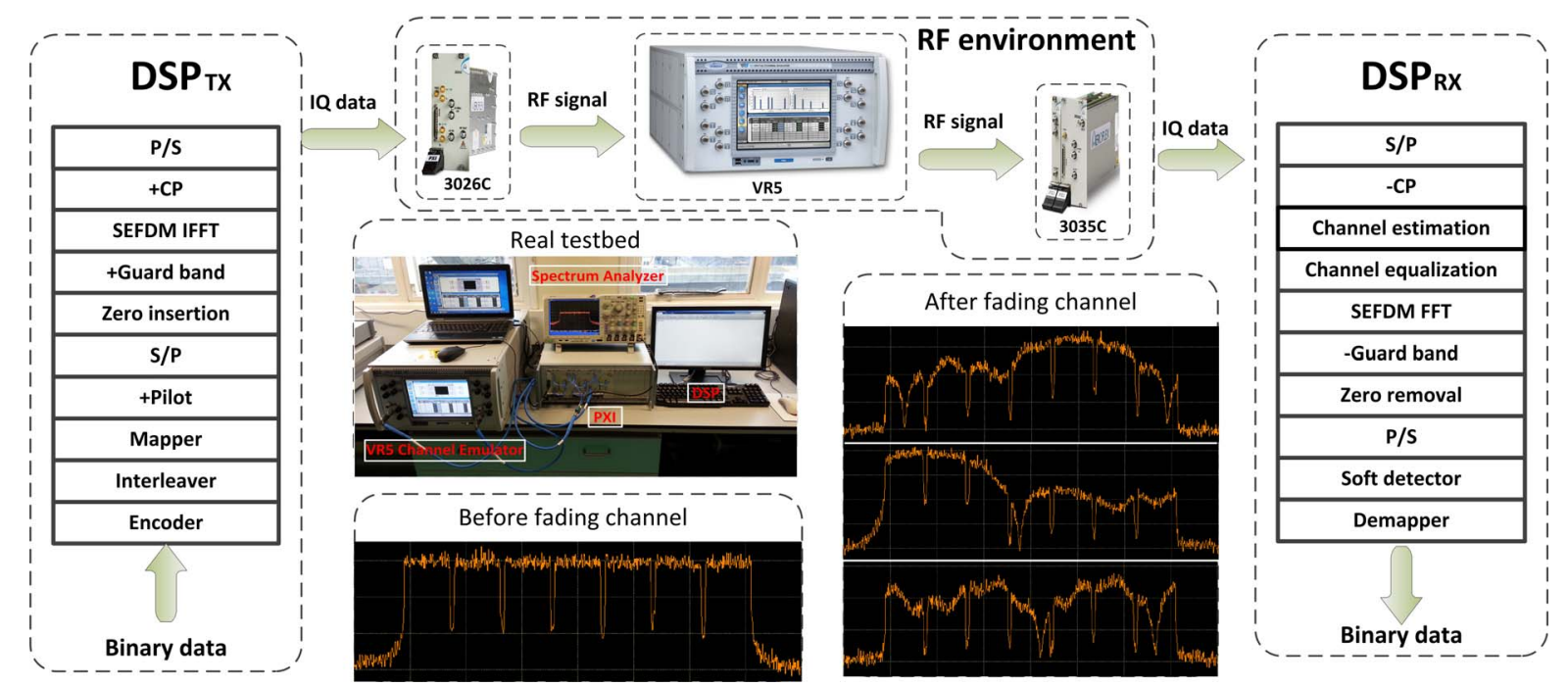

Figure 3. Experimental setup for CA-SEFDM transmission in the LTE EPA5 fading channel. Spectra shown at three randomly chosen time instants obtained to illustrate the time-variant fading channel characteristics.

1) Experimental Setup: The CA-SEFDM experimental testbed, including software and hardware, is shown in Fig. 3. The software consists of two digital signal processing (DSP) blocks for signal generation and detection, at the transmitter and the receiver, respectively. The hardware consists of an Aeroflex 3026C RF signal generator, an Aeroflex 3035C RF digitizer and a Spirent VR5 channel emulator (emulating a standard LTE wireless fading channel). Signal transmission, wireless channel and signal reception are all implemented in a realistic RF environment. The real experimental testbed is shown in the middle of Fig. 3. Sample spectra are measured and included to show the effect of multipath fading channel. Detailed testbed specifications are shown in Table I.

Table I

EXPERIMENTAL SYSTEM SPECIFICATIONS FOR CA-SEFDM

\begin{tabular}{ll}
\hline Parameters & Values \\
\hline \hline Central carrier frequency & $2 \mathrm{GHz}$ \\
\hline Sampling frequency & $61.44 \mathrm{MHz}$ \\
\hline CA channel bandwidth & $25 \mathrm{MHz}$ \\
\hline Number of CCs & $5,6,7$ \\
\hline Bandwidth of one CC & $\alpha \times 5 \mathrm{MHz}$ \\
\hline Sub-carrier baseband bandwidth & $15 \mathrm{KHz}$ \\
\hline Sub-carrier spacing & $\alpha \times 15 \mathrm{KHz}$ \\
\hline IFFT/FFT size & 4096 \\
\hline Occupied sub-carriers in one CC & 301 \\
\hline Cyclic prefix & 288 \\
\hline Modulation scheme & $4 \mathrm{QAM}$ \\
\hline Channel model & EPA fading channel $(2.698 \mathrm{~km} / \mathrm{h})$ \\
\hline Doppler frequency shift & $5 \mathrm{~Hz}$ \\
\hline Channel coding & $(7,5)$ convolutional code \\
\hline Coding rate & $R_{\text {code }}=1 / 2$ \\
\hline
\end{tabular}

At the transmitter side, input binary bits are first encoded in the encoder. Then, a random interleaver $\boldsymbol{\Pi}$ is employed to permute the coded bits. The encoder and interleaver parameters are detailed to [1]. After symbol mapping, one uncoded pilot symbol is inserted at the beginning of each subframe (i.e.
1 pilot symbol and 13 complex coded symbols) and is used to estimate channel state information (CSI) and compensate for imperfect timing synchronization and local oscillator (LO) phase offset. It should be noted that pilot tones are modulated on all sub-carriers. An LTE required protection gap is inserted between each CC after the serial-to-parallel (S/P) conversion. Then, the guard band is inserted for oversampling, IFFT is used to effect modulation and CP addition is used to combat multipath delay spread. Finally, a serial IQ data stream is uploaded into the RF environment. In the RF domain, the 3026C module converts the incoming baseband digital signal to an analogue one and up-converts the analogue signal to the $2 \mathrm{GHz}$ radio frequency. The Extended Pedestrian A (EPA) LTE defined multipath fading channel model [37] is configured in the VR5 channel emulator to evaluate the system performance in a real wireless condition.

At the receiver side, the distorted analogue signal is downconverted to baseband and transformed back to digital I and Q signals within the $3035 \mathrm{C}$ module. The captured signal is then transferred to the receiver side DSP block for offline processing. After the S/P block, the $\mathrm{CP}$ is removed from each SEFDM symbol vector. A time domain channel estimation algorithm termed FCE is employed to estimate the phase and amplitude distortions of the signal. With the estimated CSI, distorted signals are compensated. The following processing is the reverse operations of those at the transmitter side. In particular, the soft detector is designed according to section II-D and is used to recover signals from the self-created ICI.

2) Measured Results: The experiment is operated based on the system specifications presented in Table I. The total occupied bandwidth is $25 \mathrm{MHz}$ due to the limitations of available equipments. A single long IFFT is used for multiple CCs signal generation following the same principle in [38]. A real-time LTE EPA5 fading channel is configured in the VR5 
channel emulator based on the channel specifications in [37]. In the time-variant channel, SEFDM symbols could experience different phase and amplitude distortions. Therefore, a total of 6,500 SEFDM symbols are tested and average performance is calculated.

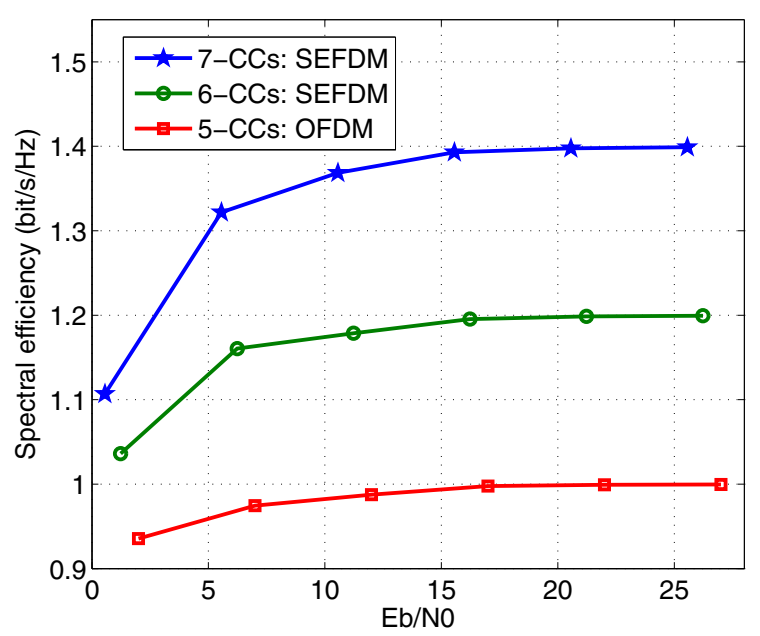

Figure 4. Effective spectral efficiency (bit/s/Hz) of different CA-SEFDM systems.

The benefit of the spectral efficiency improvement of CASEFDM signals is examined in Fig. 4 where the effective spectral efficiency is defined as the non-error bits per second per Hertz that can be achieved. The effective spectral efficiency computed in the figure is expressed as

$$
S E=\frac{1}{B} \times(1-B E R) \times R_{\text {code }} \times \log _{2} O \times N_{C C} \times R_{C C}
$$

where $B$ is the entire occupied bandwidth, $B E R$ is the bit error rate at a specific $E_{b} / N_{o}$ value, $(1-B E R)$ indicates the probability of no errors, $O$ is the constellation cardinality, $N_{C C}$ is the number of CCs ranging from 5 to 7 in the given bandwidth $B$ and $R_{C C}$ is the data rate per CC.

Clearly, the CA-SEFDM signal provides higher spectral efficiency than the CA-OFDM signal at sufficiently high $E_{b} / N_{o}$ values. As explained above, this is because extra CCs are packed in the CA-SEFDM system in a given bandwidth. It can be observed that with the increase of $E_{b} / N_{o}$, a saturation of achievable spectral efficiency is reached. As expected, the ceiling of achievable spectral efficiency for each system is variable. Generally, the relation is $S E=\frac{1}{\alpha} \times S E_{O F D M}$ where $S E_{O F D M}$ is the spectral efficiency of the OFDM based CA signal.

This dense CC packing scheme provides benefits particularly in multiuser diversity scenarios. In a wireless channel, different users have different moving speeds, hence, they experience different fading channels. Using the compressed SEFDM signal, more CCs are aggregated, each CC with a narrower bandwidth could experience a flatter channel frequency response relative to the OFDM case. In other words, the new scheme shows more flexibility in that each user may select its own preferred CCs based on their fading channel conditions.

\section{B. Millimeter Wave RoF}

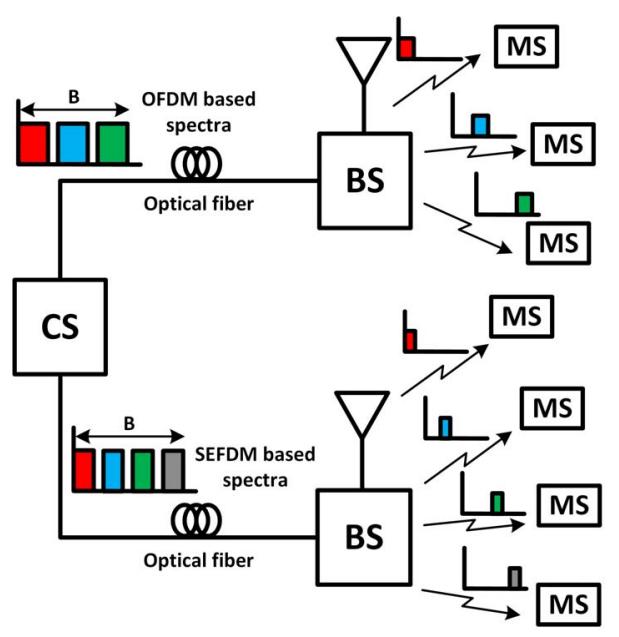

Figure 5. An application scenario of SEFDM waveform in fiber wireless networks where CS indicates central station, BS indicates base station and MS indicates mobile station.

The millimeter wave RoF scenarios for OFDM and SEFDM are illustrated and compared in Fig. 5. Spectrum (band) aggregation is considered as a way to demonstrate the difference. Signal bands are shown as blocks next to the optical fibers. For each signal band, the same number of sub-carriers is packed in the OFDM and SEFDM scenarios. It is evident that the bandwidth of each band in the SEFDM scenario is narrower than that of the OFDM scenario. The benefit is that more mobile station (MS) can be supported in a given bandwidth. In this experimental demonstration, throughput is measured and compared. Thus, one MS is assumed to occupy the entire bandwidth.

Table II

EXPERIMENTAL SYSTEM SPECIFICATIONS FOR MILLIMETER WAVE SEFDM

\begin{tabular}{ll}
\hline Parameters & Values \\
\hline \hline Millimeter wave frequency & $60 \mathrm{GHz}$ \\
\hline Intermediate frequency & $1.8 \mathrm{GHz}$ \\
\hline Baseband signal sampling frequency & $3 \mathrm{GHz}$ \\
\hline Bandwidth of baseband signal & $1.125 \mathrm{GHz}$ \\
\hline AWG sampling frequency & $12 \mathrm{GHz}$ \\
\hline Oscilloscope sampling frequency & $50 \mathrm{GHz}$ \\
\hline Length of MMF fiber & $250 \mathrm{~meters}$ \\
\hline Distance of wireless link & 3 meters \\
\hline Modulation scheme & $4 \mathrm{QAM}$ \\
\hline IFFT/FFT size & 128 \\
\hline Data sub-carriers & $48,60,80$ \\
\hline Sub-carrier baseband bandwidth & $23.4 \mathrm{MHz}$ \\
\hline Sub-carrier spacing & $\alpha \times 23.4 \mathrm{MHz}$ \\
\hline Cyclic prefix & 10 \\
\hline Channel coding & $(7,5)$ convolutional code \\
\hline Coding rate & $R_{\text {code }}=1 / 2$ \\
\hline
\end{tabular}




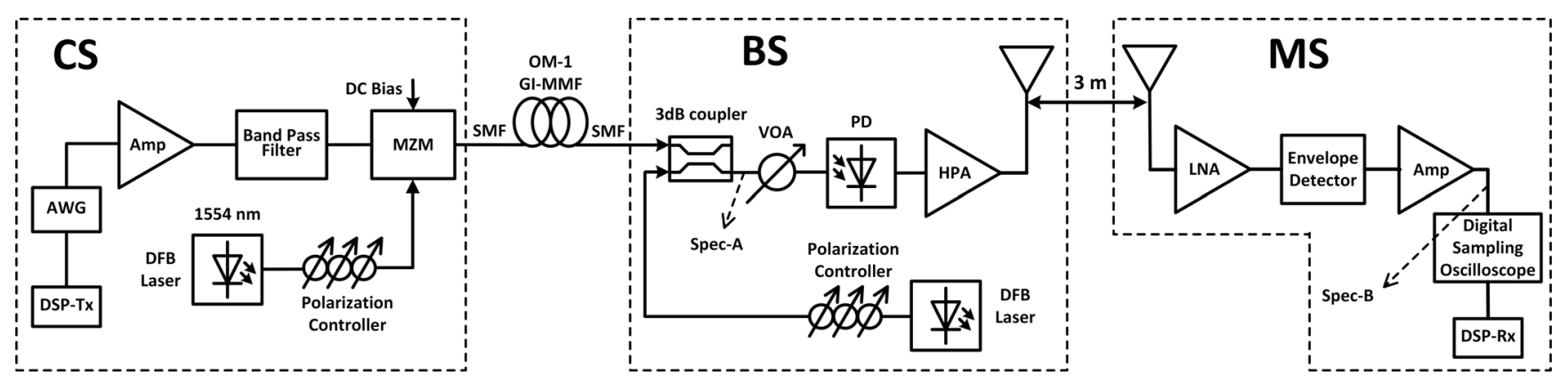

Figure 6. Experimental setup for SEFDM signal transmission over a $60 \mathrm{GHz}$ millimeter wave radio over fiber transmission link. CS indicates central station, $\mathrm{BS}$ indicates base station and MS indicates mobile station
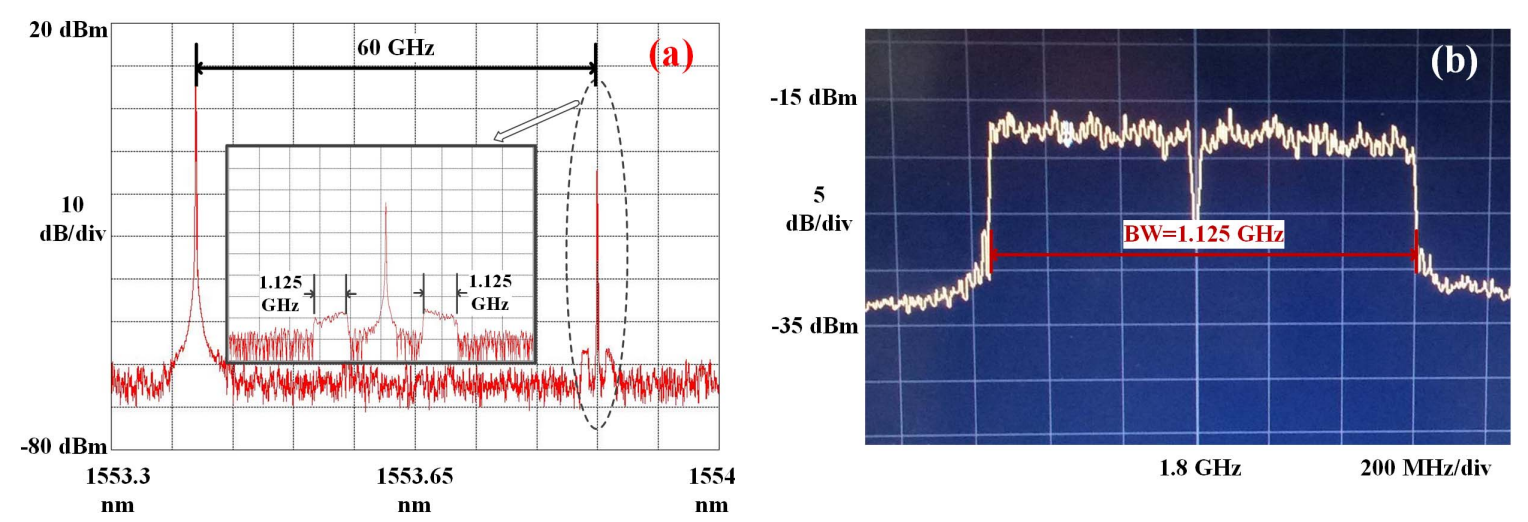

Figure 7. Optical and electrical spectra illustration. (a) indicates Spec-A in Fig.6. (b) indicates Spec-B in Fig. 6.

1) Experimental Setup: The block diagram of the millimeter wave experimental testbed is shown in Fig. 6 where both hardware and software are included. A downlink scenario is considered in this work. The software part consists of a DSPTx and a DSP-Rx. The general functions of the two blocks are similar to those in the CA experimental setup with minor modifications. One signal band is used in this experiment. Thus, the zero insertion module is removed. Moreover, in order to simplify the hardware complexity, functions such as up sampling and up conversion are moved to the DSP-Tx. Correspondingly, the down sampling and down conversion are included at the DSP-Rx.

At the central station (CS), a digital upsampled and upconverted signal from the DSP-Tx block is uploaded to a $12 \mathrm{GS} / \mathrm{s}$ Tektronix 7122B arbitrary waveform generator (AWG) for digital-to-analog (D/A) purpose. After a $20 \mathrm{~dB}$ gain amplifier and a band pass filter, the electrical signal is fed to a $3 \mathrm{GHz}$ bandwidth Mach-Zehnder modulator (MZM) and up converted to an optical frequency determined by a distributed feedback (DFB) laser at $\lambda=1554 \mathrm{~nm}$. The optical signal is delivered to the BS through a 250 meter OM-1 multi-mode fiber (MMF), coupled using single mode fiber (SMF) connectors at both ends.

The purpose of the base station (BS) is to down convert the optical signal to a millimeter wave signal. A $3 \mathrm{~dB}$ coupler is used to combine two DFB lasers. The measured optical spectrum termed Spec-A is shown in Fig. 7 (a) where signals at two wavelengths, with a $60 \mathrm{GHz}$ frequency separation, are linearly added. After a $70 \mathrm{GHz}$ bandwidth photodiode, a 60 $\mathrm{GHz}$ millimeter wave signal is generated based on the concept described in [39]. Then the amplified signal is radiated to the MS through a 3 meter point-to-point wireless channel. It should be noted that the two laser scheme is flexible in that different required carrier frequencies may be generated by adjusting the laser separation. One design principle of the $\mathrm{BS}$ is to make it as simple as possible since a number of these would be deployed in an RoF system. Considering the installation cost, the second laser can be moved from the BS to the $\mathrm{CS}$. In this case, the $3 \mathrm{~dB}$ coupler, the polarization controller and the DFB laser can be removed from the BS, which makes the system more cost effective. However, the delivered optical signal in the fiber will have a similar spectrum to Fig. 7 (a) where extra bandwidth is required.

At the MS, a waveguide coupled envelope detector is applied to down convert the $60 \mathrm{GHz}$ millimeter wave signal to $1.8 \mathrm{GHz}$ intermediate frequency (IF) due to its tolerance to phase noise impairment. Then a real time digital oscilloscope captures the IF signal shown in Fig. 7 (b) and outputs the IQ data to the DSP-Rx for offline signal processing. 


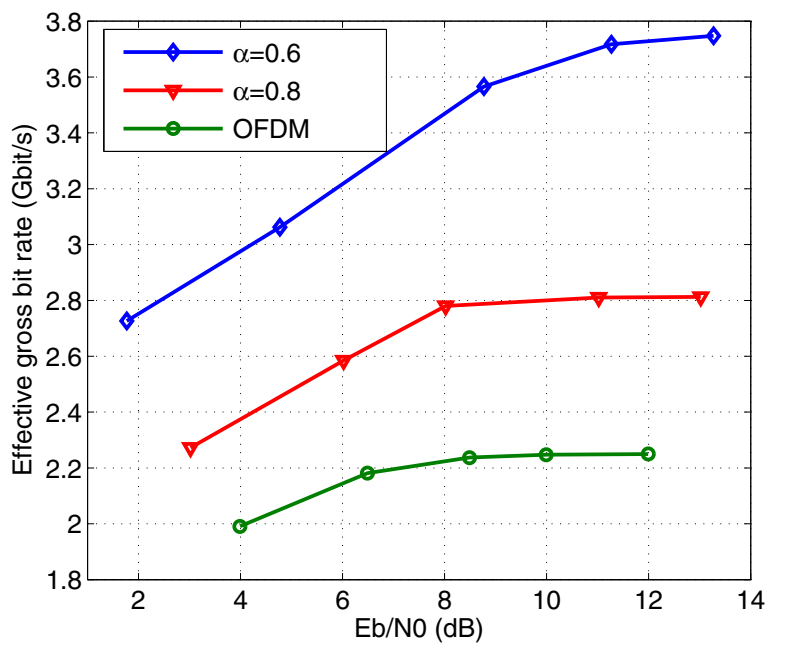

Figure 8. Effective gross bit rate of SEFDM signals at $60 \mathrm{GHz}$ millimeter wave through 3 meters wireless and 250 meters MMF fiber transmission with 4QAM symbols.

2) Measured Results: Unlike the measurement in section III-A, this experiment tests a single band, which occupies the same bandwidth for both OFDM and SEFDM. The effective gross bit rate, which is linked to BER values, is defined as the non-error bits per second that can be achieved without considering overhead, $\mathrm{CP}$ and forward error correction (FEC). Three different systems at different $E_{b} / N_{o}$ values are compared and plotted in Fig. 8 following the calculation as

$$
R_{d}=\frac{1}{\alpha} \times(1-B E R) \times B \times \log _{2} O
$$

It is apparent that the gross bit rates of SEFDM with different bandwidth compression factors are higher than those of OFDM. This is because in a given bandwidth, fixing the transmission rate per sub-carrier, more sub-carriers are packed in the SEFDM systems with smaller sub-carrier spacing. The SEFDM system with $\alpha=0.8$ can offer $25 \%=(1 / 0.8-1) \times$ $100 \%$ throughput improvement while for an SEFDM system with higher bandwidth compression and therefore higher interference, $67 \%=(1 / 0.6-1) \times 100 \%$ throughput is achieved.

\section{ApPlicAtions For Fiber Optic COMmuniCATIONS}

The LTE-like and millimeter wave SEFDM signals have been practically tested and verified through transmission over realistic wireless channels. These applications can provide the last mile communications to end users due to their supports of mobility in wireless environments. In order to deliver the broadband data service to several base stations or distribution points, optical fibers are used instead of copper cables. Henceforth, this section tests lightwave SEFDM signals to show that SEFDM signals are compatible with fiber-optic systems.

Optical systems can be categorized either as coherent or non-coherent (direct detection) systems. A coherent system leads to better performance in terms of spectral efficiency.
However, a coherent system requires the use of a receiver local oscillator and accurate phase/frequency offset estimation at the receiver, thus increasing the complexity of both the transmitter and the receiver [40]. A direct detection system may operate with a single photo-detector (PD) at the receiver, and does not require carrier frequency and phase offset estimation, due to the elimination of the local oscillator, making it well suited to cost efficient applications. However, in the direct detection scenario, a frequency gap [41] of the same bandwidth as a signal band is needed between the optical carrier and the signal band in order to combat the second-order intermodulation distortion (IMD) close to the optical carrier due to the squarelaw distortion introduced by a photodiode (PD). The drawback is that the excess bandwidth occupied by this spectrally inefficient gap cannot be used for data transmission.

In this section, two different optical system schemes are tested. An uncoded non-orthogonal $10 \mathrm{Gbit} / \mathrm{s}$ direct detection optical multicarrier system termed DDO-SEFDM [4] is experimentally demonstrated followed by a $24 \mathrm{Gbit} / \mathrm{s}$ coherent detection CO-SEFDM [5] system.

\section{A. Direct Detection}

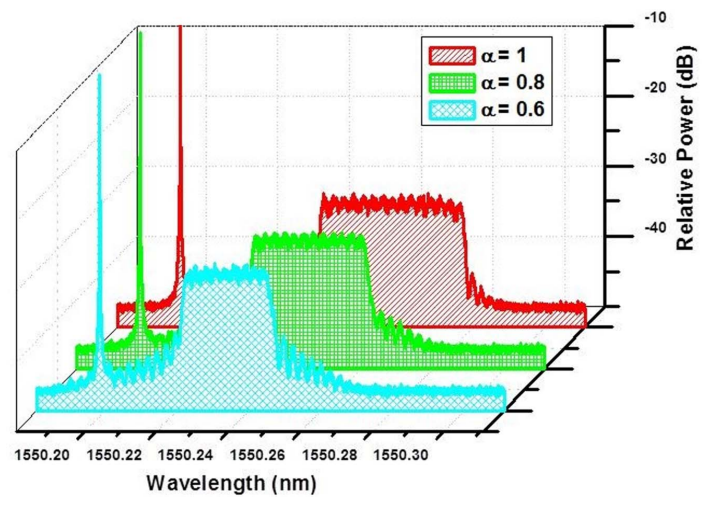

Figure 9. Experimental setup of $10 \mathrm{Gbit} / \mathrm{s}$ DOO-SEFDM system.

As shown in Fig. 9, the optical spectra of three systems are compared. The narrow high peak line in each spectrum is an optical carrier. Due to the second-order IMD introduced by a photodiode (PD), a protection gap should be reserved between an optical carrier and a signal band. The gap should be at least equal to the width of the signal band. The figure clearly shows that the frequency gap is also determined by the bandwidth compression factor. With high bandwidth compression (i.e. small $\alpha$ ), the gap can be narrowed.

In general, there are three different DDO-OFDM transmitter architectures which have been studied in [42]. The testbed complexity and signal spectral efficiency vary among these architectures. For the first architecture and the third architecture, the guard band is introduced by setting several subcarriers to zero, which is not spectrally efficient. The second transmitter architecture is preferred for the DDO-SEFDM experiment since all sub-carriers are used to transmit data. Two digital-to-analogue converters are employed with an RF 


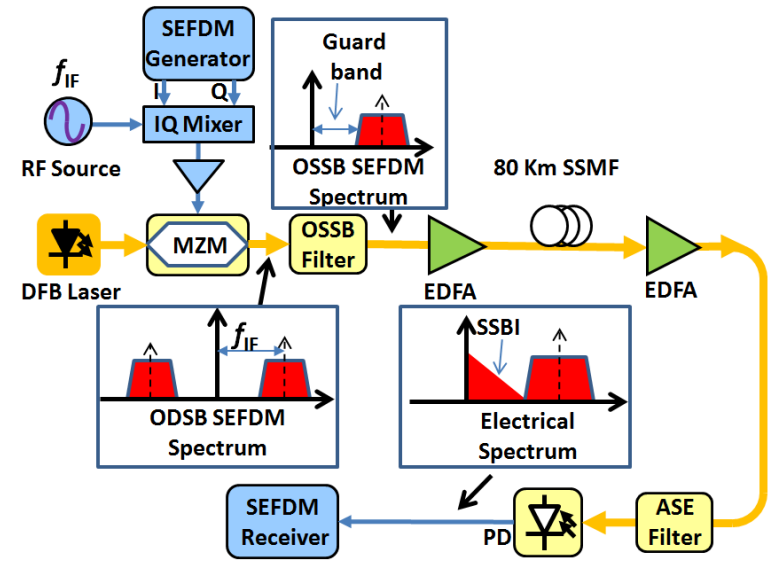

Figure 10. Block diagram of the $10 \mathrm{Gbit} / \mathrm{s}$ single sideband DDO-SEFDM experimental system.

up conversion stage. Therefore, the guard band is introduced in the RF domain by mixing complex SEFDM symbols with the RF carrier. The gap (noted as $f_{I F}$ in Fig. 10) between an optical carrier and a signal band is set to be at least equal to the bandwidth of the signal.

A baseband IQ data stream is generated in the SEFDM generator via 4QAM mapping, modulation, $\mathrm{CP}$ addition, upsampling and digital-to-analogue conversion (DAC) operations. An $\mathrm{RF}$ signal is obtained after the IQ-Mixer, which is used to up convert the baseband signal to the desired carrier frequency. A double sideband (DSB) optical signal is created after the MZM module. The optical frequency is determined by a DFB laser. An optical single sideband (OSSB) filter removes the left side band obtaining a single-sideband (SSB) optical signal. After a $80 \mathrm{~km}$ SSMF transmission link, EDFA amplification, amplified spontaneous emission (ASE) filtering and PD detection, the spectrum of the electrical signal is illustrated in the last inset of Fig. 10 together with the signal-signal beat interference (SSBI) effect. In the SEFDM receiver, a sampled data stream is obtained from a $50 \mathrm{GS} / \mathrm{s}$ Tektronix real-time oscilloscope. Then, after proper digital signal processing as shown in [4], SEFDM symbols are recovered and compared with the original transmitted ones.

The BER performance of a $80 \mathrm{~km}$ optical fiber experiment is shown in Fig. 11 where different systems are compared. The receiver employs the iterative detection-FSD (ID-FSD) [32] to cancel out the self-created interference and determine the received symbols with close to optimal error performance. The signal is formatted with 16 data sub-carriers. Channel estimation is operated by sending training symbols once at the beginning of the data stream.

There is a balance between the bandwidth compression and optical signal-to-noise ratio (OSNR) values required for a given bit error rate. In the figure, $20 \%$ bandwidth saving is achieved with around $0.7 \mathrm{~dB}$ performance degradation while for further bandwidth compression of 30\%, higher OSNR penalties are observed. It should be noted that the 4QAM

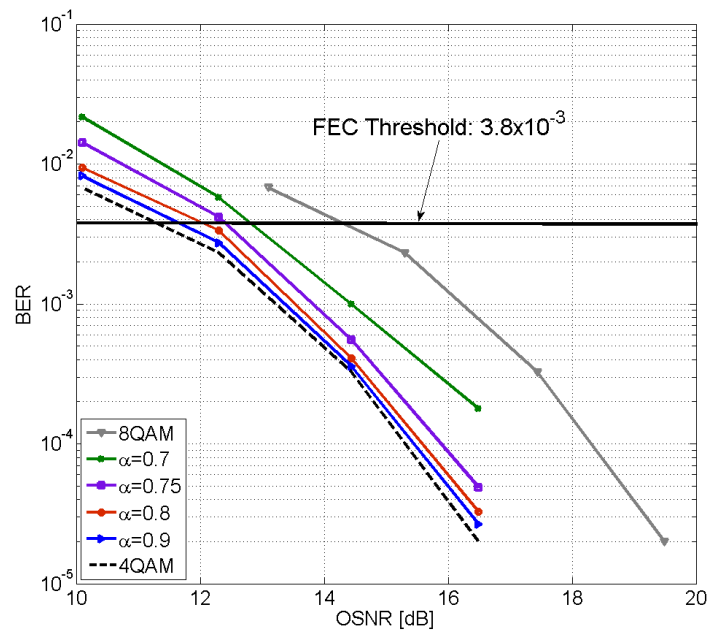

Figure 11. BER versus OSNR for $80 \mathrm{~km}$ optical fiber direct detection experiment.

SEFDM with $\alpha=0.7$ has spectral efficiency equals to $\mathrm{SE}=2.86$ $\mathrm{bit} / \mathrm{s} / \mathrm{Hz}$, which is close to that of an 8QAM OFDM whose spectral efficiency is $3 \mathrm{bit} / \mathrm{s} / \mathrm{Hz}$. At $\mathrm{BER}=3.8 \times 10^{-3}$, the 4QAM SEFDM with $\alpha=0.7$ outperforms the spectral efficiency equivalent 8QAM OFDM by around $1.5 \mathrm{~dB}$. Thus, it is experimentally demonstrated that the lower modulation format, 4QAM can replace the higher one, 8QAM of similar spectral efficiency and achieve better performance.

\section{B. Coherent Detection}

The benefit of a coherent system is its high spectral efficiency since no protection gap is reserved between the optical carrier and the signal band. Moreover, to improve further the spectral efficiency, a dual polarization transmission scheme is applied to the coherent optical-SEFDM (CO-SEFDM) experimental testbed, as shown in Fig. 12.

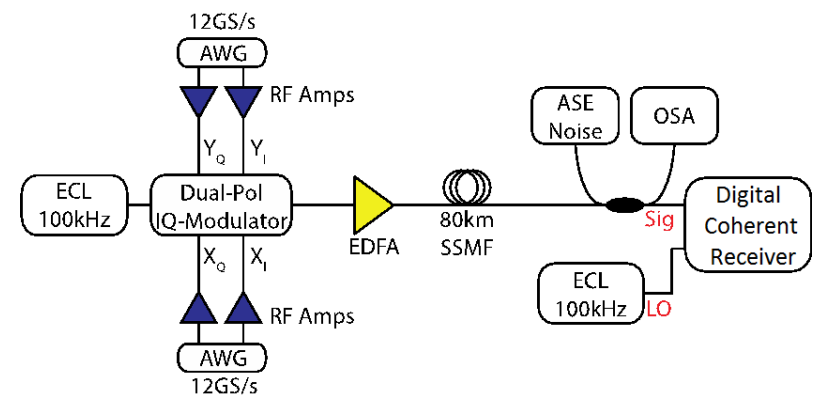

Figure 12. Experimental setup of DP-CO-SEFDM transmission system.

The benefit comes at the cost of additional digital signal processing efforts. Two external-cavity laser (ECL) diodes are used at the transmitter and receiver sides, resulting in introducing frequency offset and phase noise. In addition, due to the use of both polarizations, the polarization rotation and polarization mode dispersion (PMD) during transmission 


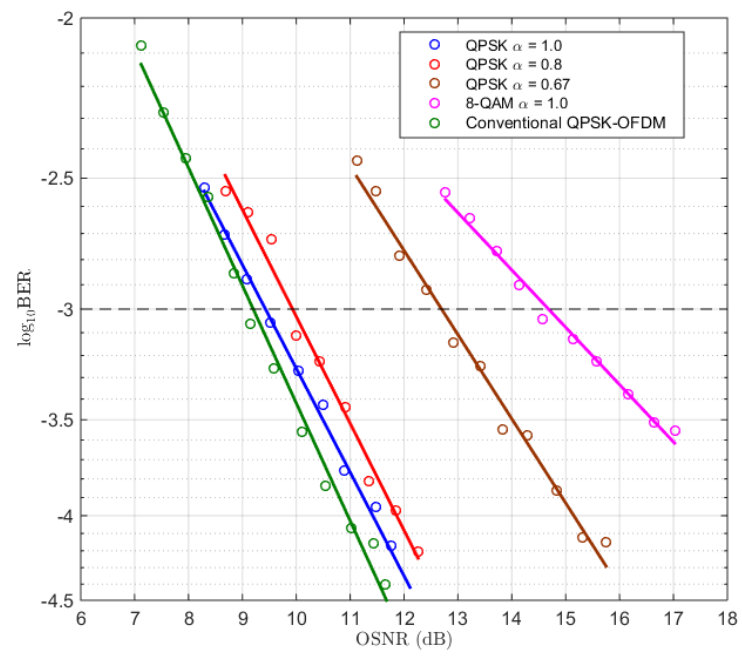

Figure 13. BER performance of $80 \mathrm{~km}$ optical fiber transmission using coherent detection testbed at various $\alpha$.

through the fiber must be solved. Therefore, the transmitted signal has to be judiciously designed and formatted. At the beginning of a data stream, a timing sequence is added for frame synchronization, followed by a training sequence for polarization corrections. Then in the RF domain, a DC pilot tone is inserted at the transmitter side, effectively translating to a fixed frequency optical carrier signal for receiver side frequency and phase offset corrections.

For each experiment two independent data signals, each occupying a predefined bandwidth ranging from $6 \mathrm{GHz}(\alpha=1)$ to $4.02 \mathrm{GHz}(\alpha=0.67)$, are generated from the two AWGs of Fig. 12. A protection guard band of $1.85 \mathrm{GHz}$ is reserved for the DC pilot insertion. The gap separates the positive and negative frequency sub-carriers leading to a simplified signal detection for each band. The formated signal is fed to an integrated dual polarization IQ modulator and up converted to an optical frequency using an ECL with a linewidth of $100 \mathrm{kHz}$. Transmitting through a $80 \mathrm{~km}$ standard single mode fiber (SSMF) link, an ASE noise source is used to vary the OSNR. The received signal is coherently detected using a polarization diverse coherent receiver, which utilized a second $100 \mathrm{kHz}$ ECL as an LO. The electrical signal is captured using a $50 \mathrm{GS} / \mathrm{s}$ real-time digital oscilloscope with a $16 \mathrm{GHz}$ analogue electrical bandwidth. Then, the DSP implementation is performed offline in MATLAB to get final recovered signals. More details about the DSP operation may be found in [5].

The experimental results for a $80 \mathrm{~km}$ optical fiber signal transmission are shown in Fig. 13. Both QPSK with $\alpha$ ranging from 1 down to 0.67 and 8QAM modulation formats are studied. It should be noted that the QPSK symbol modulated DP-CO-SEFDM with $\alpha=1$ is equivalent to a DP-CO-OFDM system. In addition, a conventional QPSK modulated OP-COOFDM system, which is assisted by periodic pilot symbols, is also tested in this work as a reference. The DP-CO-SEFDM was configured to a transmission rate of $24 \mathrm{Gbit} / \mathrm{s}$ using 16 sub-carriers. Using the same transmission bit rate, the conventional QPSK based DP-CO-OFDM is designed with 256 sub-carriers, 16 of which are reserved for pilot subcarriers.

The spectral efficiency of QPSK with $\alpha=0.67$ is equivalent to that of 8QAM. Thus, the performance comparison between them is of interest. The performance gap is around $2 \mathrm{~dB}$, which indicates that in a dual polarization coherent optical system, considering the same spectral efficiency, an SEFDM system with a low order modulation format outperforms an OFDM system with a higher order one.

\section{CONCLUSIONS}

A non-orthogonal multicarrier technique termed spectrally efficient frequency division multiplexing (SEFDM) has been practically investigated in this paper. Relative to OFDM systems, SEFDM improves spectral efficiency by reducing the sub-carrier spacing. Three experimental scenarios are described and evaluated for promising 5G network radio access and backhaul connections. We first demonstrate an enhanced carrier aggregation (CA) testbed in a realistic wireless environment. Results show that more component carriers (CCs) can be aggregated in a given bandwidth. On one hand, higher data rates are achieved. On the other hand, each user has more options to select its own preferred component carriers according to fading channel conditions within a given bandwidth indicating enhanced multiuser diversity. Then, a $60 \mathrm{GHz}$ millimeter wave SEFDM signal is investigated and practically tested in a fiber wireless environment since this frequency can support the increasing bandwidth requirement of future $5 \mathrm{G}$ networks. Finally, we show direct and coherent detection optical fiber experiments, which are targeted to offer higher data rates to backhaul connections. Our provided experimental scenarios are closely related and the combination of them may offer potential solutions for future $5 \mathrm{G}$ networks.

\section{REFERENCES}

[1] T. Xu and I. Darwazeh, "Bandwidth compressed carrier aggregation," in IEEE ICC 2015 - Workshop on $5 G$ \& Beyond - Enabling Technologies and Applications (ICC'15 - Workshops 23), London, United Kingdom, Jun. 2015, pp. 1107-1112.

[2] S. Mikroulis, T. Xu, and I. Darwazeh, "Practical demonstration of spectrally efficient FDM millimeter-wave radio over fiber systems for 5G cellular networking," in Proc. SPIE, vol. 9772, 2016, pp. 97 720I1-97 720I-8.

[3] T. Xu, S. Mikroulis, J. Mitchell, and I. Darwazeh, "Bandwidth compressed waveform for $60 \mathrm{GHz}$ millimeter-wave radio over fiber experiment," Journal of Lightwave Technology, 2016 (to appear).

[4] I. Darwazeh, T. Xu, T. Gui, Y. Bao, and Z. Li, "Optical SEFDM system; bandwidth saving using non-orthogonal sub-carriers," Photonics Technology Letters, IEEE, vol. 26, no. 4, pp. 352-355, Feb 2014.

[5] D. Nopchinda, T. Xu, R. Maher, B. Thomsen, and I. Darwazeh, "Dual polarization coherent optical spectrally efficient frequency division multiplexing," Photonics Technology Letters, IEEE, vol. 28, no. 1, pp. 83-86, Jan 2016.

[6] J. Andrews, S. Buzzi, W. Choi, S. Hanly, A. Lozano, A. Soong, and J. Zhang, "What will 5G be?" Selected Areas in Communications, IEEE Journal on, vol. 32, no. 6, pp. 1065-1082, June 2014.

[7] C. Shahriar, M. La Pan, M. Lichtman, T. Clancy, R. McGwier, R. Tandon, S. Sodagari, and J. Reed, "PHY-layer resiliency in OFDM communications: A tutorial," Communications Surveys Tutorials, IEEE, vol. 17, no. 1, pp. 292-314, Firstquarter 2015. 
[8] 3GPP TS 36.300 version 8.12.0 Release 8, "Evolved universal terrestrial radio access (E-UTRA) and evolved universal terrestrial radio access network (E-UTRAN); overall description; stage 2 (release 8)," April 2010.

[9] E. Larsson, O. Edfors, F. Tufvesson, and T. Marzetta, "Massive MIMO for next generation wireless systems," Communications Magazine, IEEE, vol. 52, no. 2, pp. 186-195, February 2014.

[10] T. Rappaport, S. Sun, R. Mayzus, H. Zhao, Y. Azar, K. Wang, G. Wong, J. Schulz, M. Samimi, and F. Gutierrez, "Millimeter wave mobile communications for 5G cellular: It will work!" Access, IEEE, vol. 1, pp. 335-349, 2013.

[11] W. Roh, J.-Y. Seol, J. Park, B. Lee, J. Lee, Y. Kim, J. Cho, K. Cheun, and F. Aryanfar, "Millimeter-wave beamforming as an enabling technology for $5 \mathrm{G}$ cellular communications: theoretical feasibility and prototype results," Communications Magazine, IEEE, vol. 52, no. 2, pp. 106-113, February 2014.

[12] B. Farhang-Boroujeny, "OFDM versus filter bank multicarrier," Signal Processing Magazine, IEEE, vol. 28, no. 3, pp. 92-112, May 2011.

[13] N. Michailow, M. Matthe, I. Gaspar, A. Caldevilla, L. Mendes, A. Festag, and G. Fettweis, "Generalized frequency division multiplexing for 5th generation cellular networks," Communications, IEEE Transactions on, vol. 62, no. 9, pp. 3045-3061, Sept 2014.

[14] M. Rodrigues and I. Darwazeh, "Fast OFDM: a proposal for doubling the data rate of OFDM schemes," in International conference on Telecommunications, vol. 3, 2002, pp. 484-487.

[15] — - "A spectrally efficient frequency division multiplexing based communications system," in Proc. 8th Int. OFDM Workshop, Hamburg, 2003, pp. 48-49.

[16] J. B. Anderson and F. Rusek, "Improving OFDM: Multistream fasterthan-Nyquist signaling," in Turbo Codes Related Topics; 6th International ITG-Conference on Source and Channel Coding (TURBOCOD$I N G), 2006$ 4th International Symposium on, April 2006, pp. 1-5.

[17] A. Barbieri, D. Fertonani, and G. Colavolpe, "Time-frequency packing for linear modulations: spectral efficiency and practical detection schemes," Communications, IEEE Transactions on, vol. 57, no. 10, pp. 2951-2959, October 2009.

[18] J. Anderson, F. Rusek, and V. Öwall, "Faster-than-Nyquist signaling," Proceedings of the IEEE, vol. 101, no. 8, pp. 1817-1830, 2013.

[19] J. Mazo, "Faster-than-Nyquist signaling," Bell Syst. Tech. J, vol. 54, no. 8, pp. 1451-1462, 1975.

[20] F. Rusek and J. B. Anderson, "Constrained capacities for faster-thanNyquist signaling," IEEE Transactions on Information Theory, vol. 55, no. 2, pp. 764-775, Feb 2009

[21] N. Ahmad, S. Kamilah Syed-Yusof, N. Fisal, K. Anwar, and T. Matsumoto, "Soft-feedback MMSE equalization for non-orthogonal frequency division multiplexing (n-OFDM) signal detection," in Smart Antennas (WSA), 2012 International ITG Workshop on, March 2012 pp. 248-255

[22] D. Rainnie, Y. Feng, and J. Bajcsy, "On capacity merits of spectrally efficient FDM," in Military Communications Conference, MILCOM 2015 - 2015 IEEE, Oct 2015, pp. 581-586.

[23] E. Dahlman, S. Parkvall, J. Sköld, and P. Beming, $3 G$ evolution: HSPA and LTE for mobile broadband. Elsevier Ltd., 2007.

[24] I. Kanaras, A. Chorti, M. Rodrigues, and I. Darwazeh, "Spectrally efficient FDM signals: Bandwidth gain at the expense of receiver complexity," in Communications, 2009. ICC '09. IEEE International Conference on, June 2009, pp. 1-6.

[25] P. Whatmough, M. Perrett, S. Isam, and I. Darwazeh, "VLSI architecture for a reconfigurable spectrally efficient FDM baseband transmitter," Circuits and Systems I: Regular Papers, IEEE Transactions on, vol. 59, no. 5, pp. 1107-1118, May 2012.

[26] M. Perrett and I. Darwazeh, "Flexible hardware architecture of SEFDM transmitters with real-time non-orthogonal adjustment," in Telecommunications (ICT), 2011 18th International Conference on, May 2011, pp. 369-374.

[27] A. Chorti, I. Kanaras, M. Rodrigues, and I. Darwazeh, "Joint channel equalization and detection of spectrally efficient FDM signals," in Personal Indoor and Mobile Radio Communications (PIMRC), 2010 IEEE 21st International Symposium on, Sept 2010, pp. 177-182.

[28] S. Isam and I. Darwazeh, "Robust channel estimation for spectrally efficient FDM system," in Telecommunications (ICT), 2012 19th International Conference on, April 2012, pp. 1-6.

[29] I. Kanaras, A. Chorti, M. Rodrigues, and I. Darwazeh, "A new quasioptimal detection algorithm for a non orthogonal spectrally efficien fdm," in Communications and Information Technology, 2009. ISCIT 2009. 9th International Symposium on, Sept 2009, pp. 460-465.

[30] _ "A fast constrained sphere decoder for ill conditioned communication systems," Communications Letters, IEEE, vol. 14, no. 11, pp. 999-1001, November 2010.

[31] T. Xu and I. Darwazeh, "Multi-Band reduced complexity spectrally efficient FDM systems," in 9th IEEE/IET International Symposium on Communication Systems, Networks \& Digital Signal Processing 2014 (CSNDSP14), Manchester, United Kingdom, Jul. 2014, pp. 904-909.

[32] T. Xu, R. C. Grammenos, F. Marvasti, and I. Darwazeh, "An improved fixed sphere decoder employing soft decision for the detection of nonorthogonal signals," Communications Letters, IEEE, vol. 17, no. 10, pp. 1964-1967, October 2013

[33] T. Xu and I. Darwazeh, "A soft detector for spectrally efficient systems with non-orthogonal overlapped sub-carriers," Communications Letters, IEEE, vol. 18 , no. 10 , pp. 1847-1850, Oct 2014.

[34] 3GPP TR 36.912, "Feasibility study for further advancements for EUTRA (LTE-Advanced)."

[35] J. Beas, G. Castanon, I. Aldaya, A. Aragon-Zavala, and G. Campuzano, "Millimeter-wave frequency radio over fiber systems: A survey," Communications Surveys Tutorials, IEEE, vol. 15, no. 4, pp. 1593-1619, Fourth 2013.

[36] S. Mikroulis, T. Xu, J. Mitchell, and I. Darwazeh, "First demonstration of a spectrally efficient FDM radio over fiber system topology for beyond 4G cellular networking," in Networks and Optical Communications (NOC), 2015 20th European Conference on, June 2015, pp. 1-5.

[37] 3GPP Evolved Universal Terrestrial Radio Access (E-UTRA) TS 36.104 V10.2.0, "Base station (BS) radio transmission and reception," May 2011.

[38] S. Bassam, W. Chen, M. Helaoui, and F. Ghannouchi, "Transmitter architecture for CA: carrier aggregation in LTE-advanced systems," Microwave Magazine, IEEE, vol. 14, no. 5, pp. 78-86, July 2013.

[39] O. Omomukuyo, M. Thakur, and J. Mitchell, "Simple 60-GHz MBOFDM ultrawideband RoF system based on remote heterodyning," Photonics Technology Letters, IEEE, vol. 25, no. 3, pp. 268-271, Feb 2013.

[40] S. Savory, "Digital coherent optical receivers: Algorithms and subsystems," Selected Topics in Quantum Electronics, IEEE Journal of, vol. 16 , no. 5, pp. 1164-1179, 2010.

[41] A. Ali, J. Leibrich, and W. Rosenkranz, "Spectral efficiency and receiver sensitivity in direct detection Optical-OFDM," in Optical Fiber Communication - incudes post deadline papers, 2009. OFC 2009. Conference on, 2009, pp. 1-3.

[42] B. Schmidt, A. Lowery, and J. Armstrong, "Experimental demonstrations of electronic dispersion compensation for long-haul transmission using direct-detection optical OFDM," Lightwave Technology, Journal of, vol. 26, no. 1, pp. 196-203, 2008 